\title{
j2eeprof - a tool for testing multitier applications
}

\author{
Paweł Klaczewski and Jacek Wytrębowicz \\ Institute of Computer Science of Warsaw University of Technology \\ P.Klaczewski@elka.pw.edu.pl,J.Wytrebowicz@elka.pw.edu.pl
}

\begin{abstract}
Quality assurance of multitier application is still a challenge. Especially difficult is testing big, distributed applications written by several programmers, with the use of components from different sources. Due to multi threaded and distributed architecture, their ability to be observed and their profiling are extremely difficult. J2eeprof is a new tool developed for testing and profiling multitier applications that run in the J2EE environment. The tool is based on the paradigm of aspect insertion. The main goal of j2eeprof is to help in fixing of integration errors and efficiency errors. This paper presents the concept of j2eeprof and gives some insides of j2eeprof development. On the beginning we give some introduction to the methods of software profiling, and a brief characteristic of existing profilers, i.e., JFluid, Iron Track Sql, OptimizeIt Server Trace and JXInsight. Next we present the architecture of j2eeprof, and we describe how it collects data, what protocols it uses, and what kind of analysis it supports. On the end we demonstrate how j2eeprof works in practice. In conclusions we list the strong and weak points of this tool, which is still in a beta version. J2eeprof is planned to be offered as an open source for the programmer community.
\end{abstract}

\section{Introduction}

Software testing and software profiling are time consuming tasks, especially during development of multitier, distributed applications. Sometimes these tasks take more time than coding. They are crucial when the target application is safety or business critical. We mean by testing the process of defect discovery in a developed code. We mean by profiling the process of performance analysis of an application.

Because Java Platform Enterprise Edition (J2EE) is a widely used programming platform for developing and running distributed multitier architecture applications, we have focused our attention on testing and profiling applications that run in the J2EE environment. The result is j2eeprof [7] - a new tool to help in fixing of integration errors and efficiency errors. Integration testing and profiling need very similar methods and tools. We shortly describe them.

To make not frequent or exceptional conditions testable we have to extend the tested application to make controllable its execution flow. During an execution flow a programmer collects selected data for subsequent analysis. Selection of the data depends on programmer aim, it could be: remote function checking, bottleneck discovery, time consumption of selected functions and memory consumption. In general, there are two methods of data gathering: sampling and tracing $\infty$. The advantage of sampling is that this method slightly influences the tested application in contradiction to the tracing 
method. The advantage of tracing is the possibility to achieve very high accuracy but when accuracy is higher - the execution time is more and more disturbed.

Extensions that make the execution flow controllable are included in the application code by a programmer. Sampling can be performed without any modification of the application code. Tracing can be achieved by altering the code or by modification of its environment, or both. The Java Platform Debugger Architecture (JPDA), which is a collection of APIs to debug Java code, is a good example of a tool for environment modification. A disadvantage of JPDA is the limited set of low-level events that the programmer can observe. The abstraction level of virtual Java machine is not suitable for J2EE application analysis. The programmer gets too much low level data, which are difficult to analyze. Altering of the application code can be done by hand, can be processed by a compiler (e.g., as for gprof Unix tool), or after compilation. There are Java libraries, e.g., BCEL ${ }^{1}$, ASM [3], which allow altering a Java bytecode during loading. The programmer has to point where and how the automatic code altering should be performed.

The amount of data collected during an application run is usually huge. Sometime some compression or aggregation methods have to be used for their collection. A programmer needs to have some tools for filtering the collected data and for their visualization in an interactive manner. G. Ammons, T. Ball and J. R. Larus [1] have proposed to build a structure called Calling Context Tree (CCT) - as an aggregation method. Every tree node keeps some measurements of an executed function. Any path in the tree represents a possible execution sequence of modules (the module can be a method, a component, a layer, or a node belonging to a distributed system). Figure 1 depicts an exemplary execution path of a function $X$ that executes 6 modules $(A B$ notation means that $\mathrm{A}$ module calls $\mathrm{B}$ module). A tree representation is more expressive.

$$
\begin{aligned}
& A \rightarrow B \\
& B \rightarrow C \\
& B \rightarrow D \\
& D \rightarrow E \\
& A \rightarrow F
\end{aligned}
$$

(a)

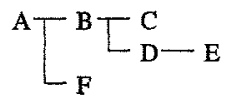

(b)

Fig. 1. Execution path visualization a) sequential, b) context tree

It helps to find bottlenecks related to different load of data or user connections. There are more ways of execution path visualization as Fig 2 shows. Nowadays profilers generate a layered representation of full tree of execution calls (Fig 2b). The width of every rectangle may depict execution time of relating module. Complex applications give very big trees. To make them more readable reduced graphs can be generated (Fig. $2 \mathrm{c}, 2 \mathrm{~d}$ ). Most profilers allow for simple filtering of presented data with predefined set of views. However there are exceptions: a programmer using ejp $p^{2}$ can implement own filters. XDSE profiler [2] stores full execution trace in an XML database. Next a programmer can define filtering by XQuery language and select a visualization form.

\footnotetext{
${ }^{1}$ http://jakarta.apache.org/bcel

${ }^{2}$ http://ejp.sourceforge.net
} 


\section{$\mathrm{M}(\mathrm{A}(\mathrm{B} O \mathrm{C}()) \mathrm{D}(\mathrm{CO}) \mathrm{A}(\mathrm{B}() \mathrm{CO})) \mathrm{D}(\mathrm{C}(0) \mathrm{A}(\mathrm{B}(\mathrm{B}(\mathrm{C}) \mathrm{C}(0))$}

(a)

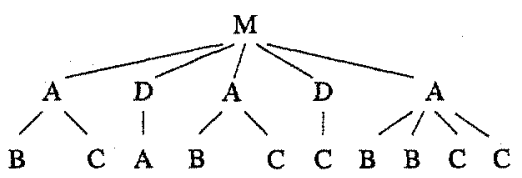

(b)

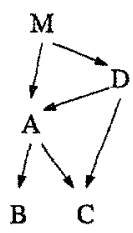

(c)

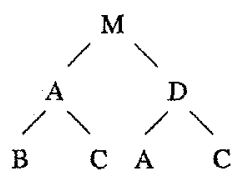

(d)

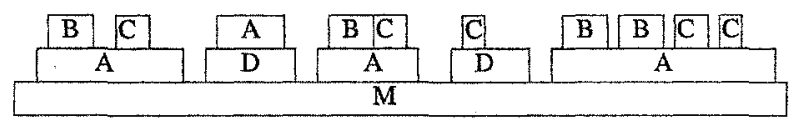

(e)

Fig. 2. Execution path visualization a) layered representation, b) full tree of execution calls, c) reduced call graph, d) context call tree. e) trace graph

Profiling of a distributed system is difficult. Every distinct element has to be observed independently. Next, a profiler has to correlate collected data before filtering and presentation. A correlation method based on independent clocks is not accurate and leads to interpretation errors. Much efficient is to include tracing into a communication mechanism used by separate instances. Authors of [8] describe a tool that traces TCP messages. For better efficiency, a profiler could use some marking of messages that concern the analyzed application/purpose. Pinpoint project [6] is based on modification of $J_{b o s s^{3}}$ application server - in this way a distributed application, which works on Jboss servers, can be easily and efficiently traced. When a programmer uses CORBA, then we can take advantage of built in interceptor mechanism for message marking. The interceptor is a function written by the programmer and called during communication.

There are several commercial profilers addressed to J2EE environment, but we do not know any such a tool from public domain. Profilers created for Java programmers, not only for those who use J2EE, are more numerous. Let take a look on some of them - the most interesting in our opinion. JFluid profiler [5], from Sun Microsystems, works only with the NetBeans programmer framework. It provides some means for analysis of: memory consumption, execution time and execution flow. Programmer can point some Java methods for analysis. JFluid process the code statically to discover all methods, which could be executed by those selected. Next it alters them to make them traceable. It visualizes only the traces that belong to the execution context of selected methods. The altered code has constant time overhead, that allows subtracting it from measured values, and present more accurate data. Because JFluid co-works with extended (tuned for it) virtual java machine it is a fast and efficient tool.

Iron Track Sql ${ }^{4}$ is a free tool for performance monitoring of java applications that interact with databases. It builds a log of every database query, its time and duration. It allows for some filtering, e.g., to register only these queries whose duration overcomes

\footnotetext{
${ }^{3} \mathrm{http}: / /$ www.jboss.org

${ }^{4}$ http://www.irongrid.com/catalog/product_info.php?products_id $=32$
} 
a defined threshold. It is based on a database proxy, which makes all required logs. The programmer has to use the p6spy driver (an element of Iron Track $S q l$ ) in place of standard jabc driver.

OptimizeIt Server Trace is a Borland profiler addressed to J2EE. It can gather data using probing or tracing. It can monitor memory consumption. With this tool the programmer can visualize execution paths as a context tree or as a full tree of execution calls. OptimizeIt presents j2ee services trace using sets of abstract words. In example word "ejb load" stands for ejb load life cycle method. Tool hides application server internal implementation of ejb load and presents it to user in simplified form. Profiling statistics are then more readable and free of unnecessary information. This feature makes OptimizeIt much more effective tool for J2EE application tracing than standard profiler. Optimizelt can point hot spots, can display execution time of every layer, e.g.: JDBC 23,68\%, JNDI $15.31 \%$, servlets and jsp $57.84 \%$, EJB 3,17\%. It can even register and visualize RMI communication.

JInspired company offers the JXInsight profiler. This tool is very similar to OptimizeIt Server Trace. The difference is that JXInsight has more functions for monitoring of database queries. It can display correlations between distributed events using CORBA interceptors. Both OptimizeIt and IXInsight are very complex and powerful tools, which allow multitier visualization of execution paths.

There are many other profilers but most of them suit only development of standard Java programs running on a single machine. They are inefficient for development of J2EE applications, which are distributed and use a server code. Usually the programmer does not know the server code (it is a black box for him). And the server code is a significant part of the application. The only corrections and optimizations, the programmer can make, are inside his code. Hence only tools like OptimizeIt and $J X I n s i g h t$ can really help to profile J2EE applications.

\section{2 j2eeprof insides}

J2eeprof is profiler designed for applications running in J2EE environment. J2EE provides variety of services. Programs work in a container i.e., servlet container or ejb container. Container provides services, can manage component life cycle and enhance program behavior. The way program uses services can be specified in code or configuration descriptor. When configuration is used it is impossible to inspect program behavior only by reading its code. This makes testing more difficult to the programmer. Another problem arises, when J2EE application is profiled using standard java profiler. There is huge amount of container implementation code execution registered together with program code. The performance impact is large and results contain plenty of superfluous information.

In order to capture accurate view of execution flow, j2eeprof uses tracing. J2eeprof comes with ability of selective program tracing. It registers J2EE services and program execution at high level of detail. By inspecting trace programmer can find out all the interactions of $\mathrm{J} 2 \mathrm{EE}$ services with program. The tool has significant ability to shape profiling scope. J2eeprof addresses also distributed nature of ejb components. It is able 
to track communication between remote ejb components and deliver distributed system trace.

J2eeprof is designed for profiling applications that run in a distributed environment. Thus tool itself is distributed as well. There are three major modules of j2eeprof: data collection module, transport module and visualization module. The data collection is installed on distributed system nodes and acts as client in the client-server j2eeprof architecture. Visualization module is responsible for trace analysis and visualizations. The data is transported from remote data collections modules to visualization module by transport module.

\subsection{Data collection}

Data collection module uses tracing method to collect profile data. Its implementation is based on the aspect oriented programming (AOP). Aspect is a program module that implements some common functionality and has no dependencies on other program modules. AOP consists of two elements: aspect weaver and composition language. Aspect weaver is responsible for composition of aspects and other modules into final application. Composition language controls the weaver. J2eeprof uses Aspectwerkz ${ }^{5}$, open source AOP library, as a basis for data collection module. Aspectwerkz weaver is capable of dynamic aspect insertion. This feature enables profiler to temporarily modify tested code and change profiling scope on every program execution. Aspectwerkz uses Aspect $J^{6}$ composition language. The point of program code, where aspect can be inserted, is called join point. It can be i.e., a method or a construction invocation. Pointcut is AspectJ definition that pick out a set of join points. Aspect I gives j2eeprof capability to define profiling scope with detail. Important feature in J2EE environment is that a join point can define interface and polymorphic execution. J2EE is specified by a set of interfaces. J2eeprof can profile application server standard services by tracing them at the interface level. This method provides the right level of abstraction. Tracing implementation details of application server not only has negative performance impact, but also has no value for the application developer, as he cannot modify server code. Still the application code can be traced with much greater detail - up to every method call.

Data collection module implements a set of aspects. Data collection aspect is responsible for registering information on program execution. AOP composition language allows mixing of aspects in order to register traces on different detail level. Data trace representation (see Fig. 3 ) in j2eeprof consists of 4 elements. PathNode is a node of trace path. PathNode can contain other PathNode in the way it make call tree. PathNode is a base class for a concrete node, which may represent method execution or distributed call. Nodes belong to an execution thread, which is represented by ThreadNode. SystemNode is a node of distributed system. System abstracts whole observed system. The representation can describe nodes on different level of abstraction.

There are 2 generic aspects that trace method executions: MainAspect that registers only method signatures and ParametersAspect that registers also parameter values. An aspect collects information about several attributes: start and end time, information on

\footnotetext{
${ }^{5} \mathrm{http} / / /$ aspectwerkz.codehaus.org

${ }^{6} \mathrm{http}: / / \mathrm{www}$.eclipse.org/aspectj
} 


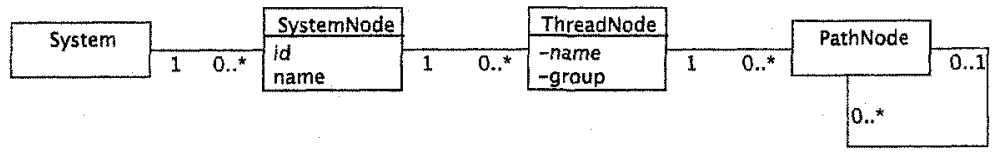

Fig. 3. Trace model

exception, path node name (e.g. method signature) and execution thread. There is also one additional attribute - category that is specified in aspect definition, and it is used later for data analysis.

\subsection{Data transport}

Gathered data are transmitted by transport module. The module consists of three parts: data sender, transport protocol and data receiver. Data transport module can write data to file or send over TCP/IP. The most important element is the protocol. J2eeprof uses binary protocol that is built in a way to keep network traffic low. We have executed several tests to measure j2eeprof overhead. The results (Table 1) have shown that the most time consuming is $\mathrm{I} / \mathrm{O}$. The more data is sent the more impact on performance is made (see test 3 and 4 in Table 1). During execution of test 3 all gathered data been discarded, during execution of test 4 the same data have been written into a file. VO slow down factor was about 6 . Addition of a simple compression method resulted in better overall performance. $J 2$ eeprof uses dictionary compression for most frequently sent data - event labels. MainAspect sends approximately 30 bytes per start method event and 22 bytes per exit method event. Executions with tracing turned off (test 1) and with AspectWerkz (test 2) empty aspect have shown a difference of performance overhead. Encoding overhead (test 3 ) is $3,232.98 \mathrm{~ns}$ but $509.68 \mathrm{~ns}$ (test 2) is the effect of using AspectWerkz and cannot be avoided. Write to the file (test 4) slows down by $17,421.38 \mathrm{~ns}$. J2eeprof performs almost twice better as $\log _{4} J^{7}$ (test 7 ). The maximum time was taken from j2eeprof statistics. It indicates that writing into a file gives more stable effects compared to sending over TCP/IP, however the second choice is much more convenient for a user.

Tab. 1. Measured performance overhead

\begin{tabular}{|l|l|r|r|}
\hline no & test & mean time[ns/per call] & max time[ms/per call] \\
\hline \hline 1 & no aspects & 31.63 & \\
\hline 2 & NullAspect & 509.68 & \\
\hline 3 & MainAspect (no I/O) & $3,232.98$ & \\
\hline 4 & MainAspect (file) & $20,654.36$ & 58 \\
\hline 5 & MainAspect (tcp local) & $33,639.00$ & 308 \\
\hline 6 & MainAspect (tcp) & $36,767.00$ & 949 \\
\hline 7 & Log4J (file) & $41,199.31$ & \\
\hline
\end{tabular}

\subsection{Distributed tracing}

J2eeprof can profile distributed J2EE systems. Execution path on each distinct node of analyzed system is recorded. But it is also required to match right local paths and

\footnotetext{
${ }^{7}$ http://logging.apache.org/log $4 j$
} 
reconstruct distributed path. Tagging messages exchanged between nodes can do this. This method has top accuracy over others, and is not affected by time differences of the nodes. EJB protocol - RMI/IIOP supports sending additional information in protocol tier, without changing interface on an application tier (Fig. 4). Corba Interceptor documentation [4] describes this feature. J2eeprof tracing mechanism can be enabled in the configuration file of application server, with no need to modify program or server code. The method is protocol dependent; j2eeprof comes with implementation for standard EJB protocol RMI/IIOP and Jboss RMI. But this solution is well suited for J2EE environment. J2EE specification requires application servers to provide transaction support and user authentication over remote calls. These services are defined in application configuration descriptors. Thus communication protocols must be able do support rpc-level communicates tagging. J2eeprof inject into EJB communication apart of transaction id and user information his own data.

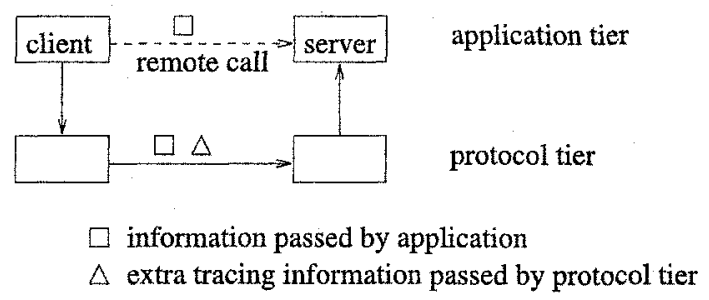

Fig. 4. Protocol tracing

Distributed paths require trace model to be improved. Model is extended by addition of two new nodes (PathNode subclasses). RPCCallNode (RC) represents an rpc call on the client side. RPCReceiveNode (RR) represents an rpc call on the server side. Figure 5 depicts reconstruction of a distributed path. On rpc call event - j2eeprof tags outgoing message with rpcld - auto generated id, unique in $\mathrm{jvm}$ scope, and node $i d$ (specified in configuration file). On rpc receive event - rpcld tag and node id are added to RR event. Node id attribute is saved in RR.sourceNodeId field. Paths merging is performed by matching RC-RR pairs. Match criteria is:

1. RC.rpcld=RR.rpcId

2. $R C$ is registered on system node defined in RR.sourceNodeld

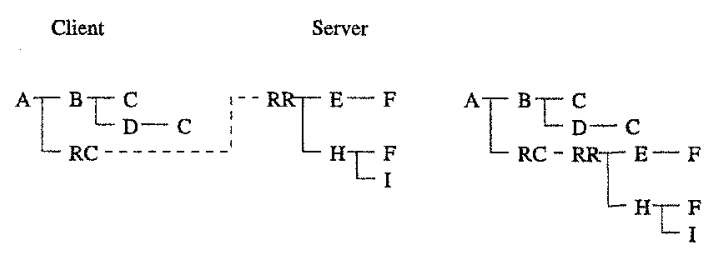

(a)

(b)

(c)

Fig. 5. Reconstruction of a distributed path a) local path on client side b) local path on server side c) completed distributed path 


\subsection{Visualisations}

J2eeprof supports several visualizations. Profiler provides data analysis on summarized trace data as well as on raw trace. Many of these visualizations are found in other tools, but distributed trace view is an original extension of them.

J2eeprof can summarize trace in form of $\mathrm{CCT}$ and flat list. Both views display total number of invocations, total, mean, minimum and the maximum execution time. CTT view provides "drill up" and "drill down" functions. "Drill up" displays all contexts in which selected node was called. "Drill down" selects all possible executions rooted in a selected node.

Raw trace can be visualized as a graph or tree. Figure 6a shows graph of a trace. The Graph is similar to tree view but every node has a rectangle form. The length and position represents execution time. For the purpose of more readable view, there is an option for displaying only top-level trace nodes (Fig. 6b). Raw trace data can be queried. The result is indicated in graph view (Fig. 6) by changing color of nodes. Raw trace views are connected each other. When user selects node in the tree list, focus in other view is set to this node.

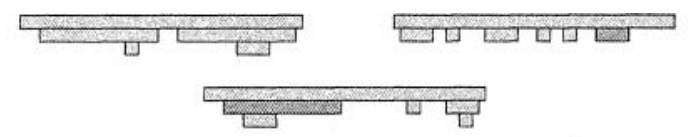

a)

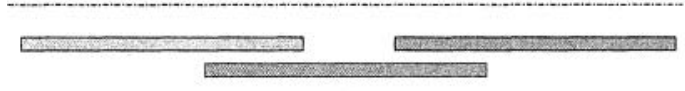

b)

filtered nodt

Fig. 6. Trace visualization a) detail, b) summary

Ability to collect distributed trace is quite uncommon in profilers. Thus there are not many ready to use solutions. Distributed trace requires special view. J2eeprof comes with original solution to this problem.

Figure 7 depicts "rpc view". The view captures distributed path on all nodes it belongs to. Apart of the path itself, the view contains also context of path on each distinct node. The view is horizontally divided in two zones. On the top, there is distributed path. On the bottom, there is context of the fragment of graph view. The view has also several vertical zones, each on every node along the distributed path. Double vertical lines divide system nodes. Doted lines mark time margin zone. In margin zone the top part of view is frozen on the contrary to the context shown in bottom part of the view. Timeline in context view is wider than in distributed path view. Thus in a case when distributed path execution of given system node is very short, still the context view show some information.

The path on Fig. 7 starts on Node 1, paths a and b. Execution of $c$ is an rpc call. That part is shown on left part of the figure. D path is executed on Node 2 - middle part of the graph. Paths a,b,c are marked with grey color as they do not belong to Node 2. Last part of the figure, on the right, displays end of paths back on Node 1. 


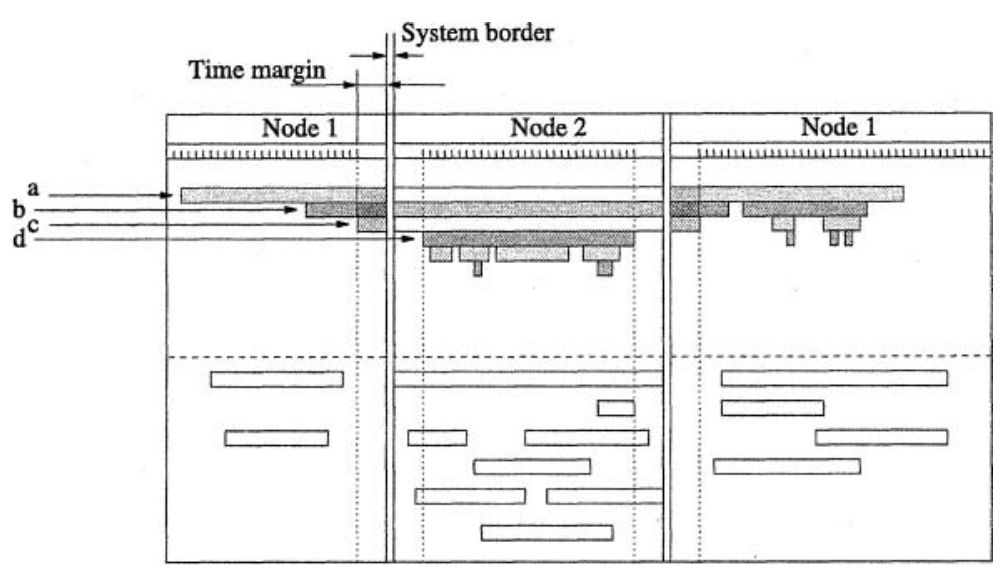

Fig. 7. Distributed trace view

\section{3 j2eeprof in practice}

J2eeprof was tested with Rubis $^{8}$ [9] - J2EE auction site benchmark. Rubis was created to compare performance of several distinct implementations of the same program. Each implementation uses different framework or technology. J2eeprof was tested with two of them. First is BMP_EntityBean_ID_BMP that is based on Entity Beans and bean managed persistence (denoted as bmp). The second EJB_CMP2 (denoted as cmp) uses Entity Beans, Session Facade design pattern and container managed persistence. Two of Rubis functions where choosen for the test. SearchItemsByCategory shows list of auction items. The second Register User registers new auction site user. These functions are very different. First one is data intensive read only function, while the second is transactional read and write function. Profiling scope included all Rubis code method calls and tracing of JDBC and JTA services on interface level. Table 2 presents performance overhead of j2eeprof in J2EE enviroment.

Test were performed on the open source application servers: Jboss 3.2 and JOnAS 4.5.3. JOnAS was configured to use iiop protocol, profiling scope included protocol tracing (using CORBA interceptors). Rubis comes with dedicated load test tool. Load tests were set to run for 5 minutes with 10 virtual users. Test where performed on AMD Athlon XP 1600+, 756RAM, Linux Slackware 10 operating system.

Rubis tests contain random factor, thus test count varies between tests. It also depends on test performance. Jonas bmp test with j2eeprof has very large overhead and test count is much lower than test without profiling. J2eeprof performance overhead factor varies from 1.1 in jboss cmp test to 56.77 in jonas bmp test. On Jboss server overhead is related only to profiling scope. Since number of registered events is reasonably small, overhead is up to $77 \%$. IIOP protocol tracing adds overhead to JOnAS test results. JOnAS tests performed slower than Jboss tests with exception of SearchItemsByCategory test (jonas cmp). The reason is that Jboss optimizes local ejb calls, JOnAS not.

Table 3 presents some insights of Rubis implementation derived from trace data. On JOnAS, bmp performed slower than cmp version but jboss cmp is slower than jboss

\footnotetext{
${ }^{8}$ Rice University Bidding System (Rubis), http://rubis.objectweb.org
} 


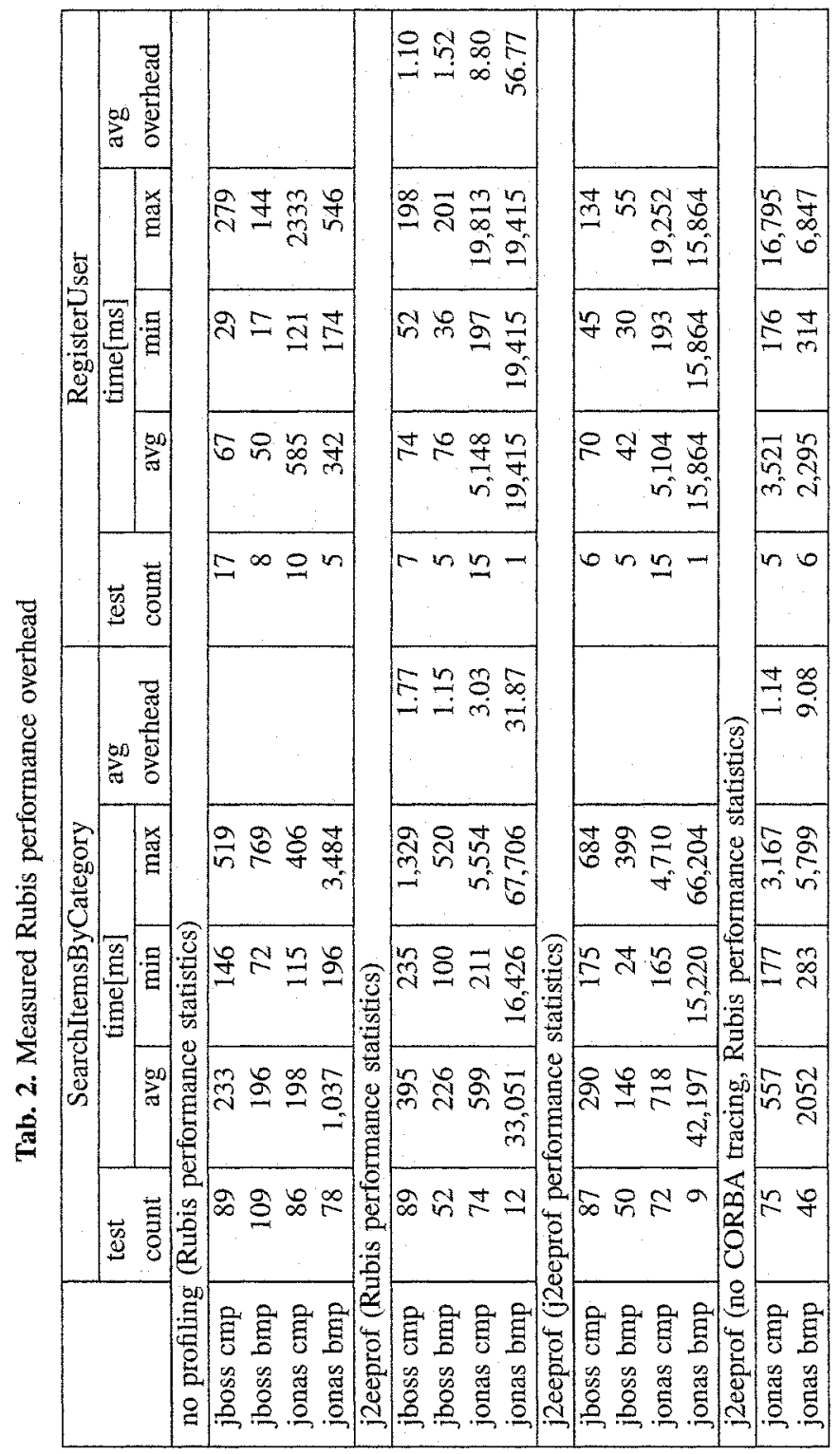


Tab. 3. Rubis tests results

\begin{tabular}{|l|c|c|c|c|}
\hline & jboss cmp & jonas cmp & jboss bmp & jonas bmp \\
\hline concurrent threads & 7 & 14 & 4 & 18 \\
\hline & \multicolumn{5}{|c|}{ SearchitemsByCategory } \\
\hline jdbc (time percent) & 51.72 & 67.84 & 27.43 & 3.93 \\
jdbc/ejb.load & 1 & 0.77 & 1 & 1 \\
jdbc/ejb.find & 1 & 0.00 & 1 & 1 \\
rmi (time percent) & 0 & 2.41 & 0 & 18.60 \\
rmi/per client call & 0 & 2 & 0 & 42 \\
\hline \multicolumn{5}{|c|}{ RegisterUser } \\
\hline jdbc (time percent) & 23.64 & 42.58 & 9.05 & 34.25 \\
jdbc calls/per client request & 11 & 6.33 & 6 & 35 \\
rmi (time percent) & 0 & 0.39 & 0 & 2.88 \\
rmi/per client call & 0 & 2 & 0 & 8 \\
\hline
\end{tabular}

bmp. Bmp Rubis implementation calls ejb entity components within web tier that results in large number of remote calls. Such design is described as J2EE anti-pattern. Jboss optimizes such calls but on JOnAS there is a remarkable average overhead of rmi call - $108.975 \mathrm{~ms}$. Cmp version uses better design - Session Facade that minimizes remote calls, there are only $2 \mathrm{rmi}$ calls in SeachItemsByCategory compared to $42 \mathrm{in}$ bmp test.

The most efficient jdbc use is done by jonas cmp version. Each pair of ejb.find and ejb.load methods result at most one jdbc call. JOnAS probably makes use of cache since jdbc calls are performed only in $77 \%$ of ejb.find calls in SearchItemsByCategory test. Other Rubis versions does not perform jdbc optimization, every ejb.load and ejb.find call results in jdbc.call. Despite of jonas cmp efficiency, the best performer is jboss bmp. JOnAS and Jboss differ also in number of observed threads. Jboss delegates one thread to a server client request so number of concurrent threads is equal to number of concurrent requests. JOnAS passes control to different thread in every rmi call. The protocol tracing mechanism is necessary to obtain complete paths in such case, although significantly increases performance overhead.

\section{Conclusions}

The purpose of j2eeprof is to help in testing and profiling of $\mathrm{J} 2 \mathrm{EE}$ distributed applications. Using it a programmer can easily analyze interactions between his code and other components or environment. Programmer does not have to modify his code to gather data. J2eeprof uses RMU/IIOP to mark and trace communication messages - giving accurate data about interactions between distributed components. The programmer decides on which abstraction level he wish to analyze his code, then he controls the trace information using aspectwerkz library. The advantage of the aspect approach is, that the programmer can easily monitor the interactions between his code and a J2EE server code. The strong features of j2eeprof are: flexibility in use, ability to fit gathered data to programmer needs, and high accuracy of registered traces from distributed components.

A week feature of jeeprof is remarkable and varied execution time overhead. All profilers that work on tracing basis, in place of sampling basis, have this disadvantage. Because j2eeprof gathers full execution trace with programmer-defined data, not just 
execution statistic, the overhead is higher than other profilers put in. To obtain accurate time characteristics, the programmer has to take other profiler that works on sampling basis. J2eeprof is small and simple tool comparing with commercial Optimizelt and JXInsight profilers. Although it is free, easy to use and we find it very useful.

\section{References}

1. G. Ammons, T. Ball and J. R. Larus: Exploiting hardware counters with flow and context sensitive profiling. In Proceedings of the SIGPLAN' 97 Conference on Programming Language Design and Implementation,pages 85-96, Las Vegas, 1997.

2. C. Anslow, S. Marshall, R. Biddle, J. Noble and K. Jackson: Xml database support for program trace visualization. In Australian Symposium on Information Visualization, volume 35, 2004.

3. E. Bruneton, R. Lenglet and T. Coupaye: Asm: a code manipulation tool to implement adaptable systems. In Adaptable end extensible component systems, Grenoble, France, 2002.

4. Interceptors Published Draft with CORBA $2.4+$ Core Chapters, Document Number ptc/2001-03-04. http://www.omg.org/cgi-bin/doc?ptc/2001-03-04

5. M. Dmitriev: Design of jffuid: A profiling technology and tool based on dynamic bytecode instrumentation. Technical report, Sun Microsystems, Nov. 2003.

6. E. Kiciman: Pinpoint: Status and future directions. 2003 www.stanford.edu/ emrek/pubs/ roc-retreat-2003-pinpoint.pdf

7. Paweł Kłaczewski: Testability Issues of Multitier Applications (in polish). Master thesis, Institute of Computer Science of Warsaw University of Technology, 2005.

8. Marcos K. Aguilerai, Jeffrey C. Mogul, Janet L. Wiener, Patrick Reynolds and Athicha Muthitacharoen: Performance debugging for distributed systems of black boxes. In Proceedings of SOSP, Bolton Landing, NY, Oct. 2003.

9. E. Cecchet and A. Chanda and S. Elnikety and J. Marguerite and W. Zwaenepoel: Performance Comparison of Middleware Architectures for Generating Dynamic Web Content, 4th ACM/IFIP/USENIX International Middleware Conference, Rio de Janeiro, Brazil, June, 2003. 